Questions vives

\section{Questions Vives}

Recherches en éducation

Vol. $6 n^{\circ} 18$ | 2012

Les recherches sur les pratiques enseignantes efficaces

\title{
Place et opportunité des travaux sur l'effet maître dans les recherches en pédagogie universitaire
}

Importance and opportuneness of studies concerning teacher effects in university pedagogy

\section{Véronique Bedin et Dominique Broussal}

\section{Q OpenEdition}

Journals

\section{Édition électronique}

URL : http://journals.openedition.org/questionsvives/1170

DOI : $10.4000 / q u e s t i o n s v i v e s .1170$

ISBN : 978-2-8218-1394-6

ISSN : $1775-433 \mathrm{X}$

Éditeur

Université Aix-Marseille (AMU)

Édition imprimée

Date de publication : 15 décembre 2012

Pagination : 111-128

ISSN : $1635-4079$

\section{Référence électronique}

Véronique Bedin et Dominique Broussal, «Place et opportunité des travaux sur l'effet maître dans les recherches en pédagogie universitaire », Questions Vives [En ligne], Vol.6 $n^{\circ} 18$ | 2012, mis en ligne le 15 octobre 2013, consulté le 10 décembre 2020. URL : http://journals.openedition.org/questionsvives/ 1170 ; DOI : https://doi.org/10.4000/questionsvives.1170

Ce document a été généré automatiquement le 10 décembre 2020.

\section{c) (i) $९$}

Questions Vives est mis à disposition selon les termes de la licence Creative Commons Attribution Pas d'Utilisation Commerciale - Pas de Modification 4.0 International. 


\title{
Place et opportunité des travaux sur l'effet maître dans les recherches en pédagogie universitaire
}

\author{
Importance and opportuneness of studies concerning teacher effects in \\ university pedagogy
}

Véronique Bedin et Dominique Broussal

\section{Introduction}

Cet article se propose de questionner la notion d'« effet maitre", lorsqu'elle est contextualisée à l'enseignement universitaire et, plus largement, d'interroger également l'efficacité de cet enseignement par le biais des recherches menées sur la pédagogie universitaire. Une telle migration peut surprendre: en effet, les recherches qui concernent l'effet maître relèvent très clairement du champ scolaire. Il nous faut expliquer ce détournement! Le choix d'importer cette notion, afin de conduire une réflexion sur la recherche en pédagogie universitaire, résulte du raisonnement suivant. Commençons par rappeler le rôle de plus en plus important tenu par les indicateurs de qualité, d'efficacité, de pertinence dans le pilotage des établissements supérieurs. Or il apparaît, paradoxalement, qu'alors même que l'université abrite en son sein une population formée et dédiée à la recherche en éducation, peu de travaux portent sur la responsabilité de l'interaction pédagogique ou de l'intervention magistrale dans la réussite des étudiants. On peut s'étonner de cette situation. En l'absence de modèle consistant, il nous est donc paru intéressant de convoquer ce pan de la recherche en éducation scolaire. Nous formions, ce faisant, l'hypothèse selon laquelle la prise en compte des travaux portant sur l'effet maitre en contexte primaire devrait permettre d'identifier des objets, des spécificités, des problématiques originales en contexte universitaire. La méthode que nous avons dès lors empruntée a consisté à mobiliser l'appareil théorique et méthodologique existant pour analyser les textes de 
communications effectuées dans le cadre de colloques de l'Association internationale de pédagogie universitaire (AIPU). Nous nous sommes attachés, dans cette analyse, à apprécier l'attention prêtée à des phénomènes relevant de l'effet maître et, dans une perspective élargie, à l'efficacité de l'enseignement universitaire. La migration se révèlet-elle féconde ? Permet-elle d'identifier des zones délaissées ? Suscite-t-elle des questions à approfondir?

2 Nous discuterons, dans le premier temps de ce texte, de la place particulière qu'occupe l'effet maître dans la recherche en pédagogie universitaire, elle-même peu développée en sciences de l'éducation en France. Dans la deuxième partie, nous interrogerons l'opportunité d'utiliser le concept d'« effet maître », quelque peu provocateur, dans ce domaine de recherche. Nous présenterons, dans les troisième et quatrième parties, la méthodologie de l'analyse conduite et les conclusions qui découlent de l'interprétation des résultats.

\section{L'effet maître : un point aveugle de la recherche en pédagogie universitaire}

\subsection{Un concept novateur pour la recherche pédagogique mais qui reste circonscrit au champ scolaire}

3 Le point de départ de la réflexion porte sur des considérations d'ordre lexicologique, donc sémantiques et herméneutiques, qui sont déterminantes dans la construction d'un objet de recherche, la production de la connaissance scientifique et sa diffusion. L'évolution du savoir passe par celle du langage, comme l'écrivent Stengers et Schlanger (1989, pp. 24-57), en stigmatisant le « pouvoir des concepts » dans l'invention scientifique. Avant d'aborder la question de la traduction du concept d'« effet maître » à l'enseignement universitaire - la traduction n'est pas un transfert -, nous allons en proposer, dans un premier temps, une définition dans le champ dont il est originairement issu, l'enseignement scolaire. Nous faisons l'hypothèse que le choix provocateur de ce concept constitue un aiguillon pertinent pour amorcer une réflexion scientifique et praxéologique sur les pratiques d'un enseignement universitaire efficace, en France notamment. Nous reviendrons largement sur ce point dans le deuxième chapitre de cet article.

«L'effet maître » constitue indubitablement un concept produit par et pour la recherche pédagogique qui vise à décrire, expliquer et comprendre spécifiquement l'effet des pratiques enseignantes sur les apprentissages des élèves. Le système d'analyse produit est majoritairement limité à l'enseignement primaire et secondaire.

5 Le projet heuristique est ambitieux car d'autres effets "maitrisables " du système éducatif pèsent dans la réussite des élèves, dont l'effet établissement et/ou l'effet classe notamment, difficiles à dissocier quelquefois de l'effet maitre lui-même. L'effet maître serait néanmoins plus fort que l'effet établissement, une fois neutralisés les effets de composition liés au public accueilli, tout particulièrement pour le début de la scolarité, comme le montrent les travaux «processus - produit» qui ont fait suite aux études multivariées visant à isoler la variable « enseignant » et son impact.

Dans une perspective plus intégrative et régulatrice, Bressoux (2001) considère que l'effet maître nécessite d'être analysé comme le produit d'une interaction maître élèves. L'efficacité des enseignants ne serait plus, dès lors, réductible à des facteurs qui leur 
seraient exclusivement propres (jugements, attentes, choix éthiques, genres et styles professionnels, type d'autorité...), même si l'action du maître demeure essentielle dans les progrès réalisés par ses élèves. Cette approche rejoint celle de Talbot (2008, p. 83) qui avance "l'hypothèse de la différenciation pédagogique et didactique selon le statut scolaire des élèves »; l'auteur s'interroge alors sur la gestion concrète, par les enseignants, de l'hétérogénéité des élèves dans leurs classes (Talbot, 2011), l'enjeu étant de favoriser la progression des plus faibles.

7 Au total, l'intérêt du concept d'« effet maitre » réside dans le fait qu'il permet de s'extirper d'une culture de la fatalité sociale, au profit de celle d'une responsabilité pédagogique élargie. Il s'intéresse à la réussite in situ des élèves, un défi qui mobilise au premier chef la recherche en sciences de l'éducation.

\subsection{Une recherche française en sciences de l'éducation en reste sur la thématique des pratiques pédagogiques universitaires}

8 Avant d'aborder des considérations d'ordre strictement scientifique, il convient, dans un premier temps, de s'intéresser à la réalité concrète des pratiques pédagogiques à l'université, L'expression est ouverte et intègre les sujets d'intérêts qui sont les nôtres : les effets des pratiques enseignantes sur les apprentissages des étudiants, sur leur échec ou leur réussite, sur l'efficacité de l'enseignement notamment. Nous limiterons le constat à l'échelle nationale: la pédagogie est le parent pauvre des activités professionnelles réalisées par les enseignants-chercheurs, au profit de celles de recherche. Ces dernières sont symboliquement mieux reconnues et plus porteuses en termes d'avancement de carrière. Les résultats des travaux des chercheurs convergent à cet égard (Dejean, 2002; Viry, 2006; Bart, 2008; Bedin \& Broussal, 2011 ; Bedin, 2012). Le principe de liberté de l'enseignement supérieur pèse également de tout son poids dans une communauté professionnelle marquée historiquement par le principe du jugement par les pairs (Mérindol, 2008) - dignus est intrare - et qui peut dès lors réagir vigoureusement à toute forme d'injonction - réforme, conseil pédagogique, etc. - (Bedin \& Saint-Jean, 2013). Audelà des pratiques et du positionnement des enseignants-chercheurs, notons également que l'existence institutionnelle de la pédagogie universitaire en est réduite à sa plus simple expression (Adangnikou, 2008). Les services universitaires de pédagogie sont très peu développés en France, «la formation continue » des enseignants-chercheurs s'opère sur le tas et informellement.

9 Ce déficit réel des pratiques pédagogiques universitaires, professionnelles ou institutionnelles, a bien évidemment un impact sur les recherches qui pourraient être conduites sur l'effet maître et sur l'efficacité de l'enseignement. Comment développer une activité scientifique sur une réalité qui n'est déjà pas posée en tant que telle? Comment conceptualiser un sujet tabou, une question sensible et finalement presque interdite à l'investigation? Peut-on transposer les modèles et les méthodes utilisés dans les autres pays?

10 Force est de constater que l'effet maître constitue bien un point aveugle de la recherche dans notre pays, et en sciences de l'éducation de surcroit. Le champ de recherche sur la pédagogie universitaire reste effectivement marginal si l'on se réfère à l'ensemble des thématiques de recherche proposées dans les équipes de recherche françaises (enquête $\mathrm{AECSE}^{1,}$ 2011) ou aux informations auxquelles permet d'avoir accès une cartographie des 
revues scientifiques du champ des sciences de l'éducation (groupe de réflexion $\mathrm{AECSE}^{2}$, 2011).

11 Si l'on considère néanmoins qu'il existe un embryon de recherche sur la pédagogie universitaire, elle est de toute façon récente, contrairement aux pays anglo-saxons ou francophones qui alimentent leurs productions depuis une trentaine d'années à partir justement de leurs propres structures d'accompagnement à l'enseignement. Les Actes des colloques de l'Association internationale de pédagogie universitaire (AIPU) reflètent cette tendance et soulignent une nouvelle fois les spécificités nationales.

Les publications de sciences de l'éducation, en France, sur les pratiques pédagogiques à l'université ont surtout vu le jour à partir des années 2000. Nous pouvons citer ceux initiés par Bru et les chercheurs de son équipe (2004) à l'Université de Toulouse2-Le Mirail (France) et par Fave-Bonnet et al. (2004). L'hétérogénéité des pratiques est soulignée ainsi que leur bricolage adaptatif individuel. L'exigence méthodologique est également rappelée, dans un domaine où les dérives "pédagogistes » et normatives constituent un risque permanent.

Les recherches plus récentes sur la pédagogie universitaire n'abordent pas pour autant la question de l'effet maître. Le dossier coordonné par De Ketele en 2010, par exemple, et qui se présente comme un bilan des recherches passées et présentes sur la pédagogie universitaire n'explique finalement que peu les effets des pratiques pédagogiques. Au total, « rares sont les approches qui appréhendent le travail universitaire dans toutes ses dimensions et dans sa variété » (Musselin, 2008, p. 5).

Un constat s'impose : la recherche en sciences de l'éducation en France, pour des raisons historiques et socio-politiques, a visiblement manifesté toujours plus d'intérêt à l'égard de la pédagogie scolaire qu'universitaire, laissant par conséquent le champ libre à d'autres disciplines (sociologie, sciences politiques...) pour explorer le domaine. En témoigne le nombre important de sociologues qui ont adhéré au Réseau d'étude sur l'enseignement supérieur (RESUP) pour illustrer le propos.

15 Si nous nous étions référés à des travaux qui dépassent le cadre national, le bilan serait différent (Boyer, 1990 ; Pelletier, 2006 ; Saroyan et al., 2006 ; Romainville, 2009 ; Langevin, 2009 ; Rege Colet et al., 2011).

\section{L'opportunité et les enjeux de l'usage du concept d'« effet maître » dans la recherche pédagogique sur l'université}

\subsection{Préalable}

16 Si l'expression "effet maître » est largement usitée dans la recherche pédagogique relative à l'enseignement primaire depuis une trentaine d'années en Europe, le terme disparaît, par contre, du vocable caractéristique de la recherche en sciences de l'éducation, dès que le périmètre s'étend à l'université française, comme nous l'avons souligné. L'enquête que nous coordonnons dans cet article le montre également sans équivoque et fait suite aux conclusions antérieures d'autres auteurs ${ }^{3}$ tels que Musselin, Fave Bonnet, Romainville, Dejean, Rege Colet notamment. Le constat de ce déficit interpelle et mérite que l'on s'y attarde. Il s'agit bien d'un point aveugle, qu'il faut d'abord rendre visible avant de s'interroger sur les raisons de cet état de fait et trouver 
éventuellement les moyens d'y remédier. La réflexion est par conséquent menée en creux et trouve finalement plus son intérêt dans ce qui n'est pas nommé - et que nous considérons comme un véritable enjeu de signification - que dans ce qui serait donné immédiatement à voir. De ce point de vue, le contenu de l'article n'est pas «conventionnel» et pose d'ailleurs un certain nombre de questions d'ordre méthodologique (l'accès à l'information, le statut des résultats, etc.).

Notre objectif n'est pas ici de faire l'apologie du concept d'« effet maître ", seulement d'essayer de réfléchir à sa pertinence dans la perspective de rendre plus intelligible la responsabilité pédagogique des enseignants du supérieur dans la qualité de l'enseignement universitaire. Les termes d'« opportunité » et d'« enjeux » utilisés dans le titre du chapitre sont explicites au regard de cette perspective.

\subsection{De l'intérêt de l'usage du concept d'« effet maître »}

18 Même si des résistances culturelles persistent (Viry, 2006), des évolutions structurelles (réformes, pressions centrifuges, etc.) sont en cours qui induisent des changements quant à la prise en compte de ce qui pourrait s'apparenter à un effet maître dans la manière d'appréhender l'évolution de l'enseignement universitaire. L'augmentation et l'hétérogénéité des publics étudiants, l'évolution rapide des technologies, la professionnalisation des formations, le développement de la formation tout au long de la vie et de l'autoformation, l'obligation d'évaluer les enseignements, pour ne citer que ces quelques facteurs, créent un véritable changement paradigmatique (Albero, 2003) qui questionne, a minima, la pédagogie universitaire et le rôle plus spécifique que les pratiques enseignantes pourraient y jouer.

Pourquoi la recherche en pédagogie universitaire serait-elle exempte de l'effet maître, alors même que ces pratiques sont confrontées au défi de leur pertinence et de leur efficacité, dans le débat actuel, international et européen, sur la qualité pédagogique de l'enseignement supérieur. La mise en œuvre du New public management, du Processus de Bologne, de la Stratégie de Lisbonne, de l'espace européen d'enseignement supérieur, nécessite de poser le débat en ces termes en faisant d'ailleurs de l'évaluation un nouvel outil managérial universitaire d'appréciation des performances des universités (FaveBonnet, 2003). La réflexion reste cependant limitée à ce que l'on pourrait nommer un " effet établissement", plutôt malencontreusement médiatisé par des classements et palmarès multiples et variés.

20 Le terme d'« effet maître " présente l'avantage de mettre l'accent sur l'efficacité pédagogique de l'enseignement et de responsabiliser les enseignants-chercheurs à cet égard. Il soulève indubitablement la question sensible de l'obligation des compétences, voire de résultats, dont on ne comprendrait pas pourquoi l'enseignement supérieur serait exclu, alors que l'orientation des étudiants, la poursuite de leurs études, leur insertion professionnelle constituent des problèmes d'actualité brûlants. Les enseignants du supérieur, pour la majorité d'entre eux, ne se nomment-ils pas " maîtres de conférences " dans l'université française? Du maître d'école au maître de conférences, de l'école à l'université, quel est l'obstacle réel et épistémologique qui gêne, voire empêche le développement d'une recherche sur l'effet maître à tous les niveaux d'enseignement? Les réformes structurelles auxquelles nous avons fait référence supra font émerger de nouvelles obligations et difficultés pédagogiques, des évolutions dans le processus d'enseignement-apprentissage dont il serait intéressant et utile que la recherche puisse 
se saisir. La centration sur les compétences des étudiants et leurs acquisitions est devenue une priorité; elle conduit à s'orienter vers un modèle pédagogique plus socioconstructiviste, au détriment du modèle transmissif traditionnel centré sur l'enseignant-chercheur et les contenus d'enseignement. Cette transformation importante demanderait à être scientifiquement analysée pour permettre, le cas échéant, de développer des pratiques d'accompagnement pédagogiques mieux adaptées.

\subsection{De l'effet maître à l'efficacité de l'enseignement universitaire}

21 Le terme d' " efficacité ", plusieurs fois employé dans ce texte, n'est pas réductible à une définition techniciste et dépasse par conséquent l'analyse stricte du « lien de conformité entre des objectifs visés [...] et des résultats effectivement obtenus. » (Bouchard \& Plante, 2002: p. 230). Dans le cas d'une recherche menée en sciences de l'éducation sur la pédagogie universitaire, nous retenons une vision plus anthropologique de l'efficacité - et de la qualité - (Behrens, 2007), centrée sur l'agir des acteurs, la complexité de leurs représentations et de leur système de valeurs, la variabilité des contextes de réalisation. L'enquête, que nous avons conduite infra, confirme cette perspective. Pour des raisons méthodologiques sur lesquelles nous reviendrons dans la suite de l'article, nous nous intéresserons à une efficacité de type interne qui met en relation des caractéristiques pédagogiques avec leurs effets sur les postures, les apprentissages (contenus et processus), les compétences des étudiants, tels que les acteurs concernés par la relation pédagogique les perçoivent eux-mêmes. Rien ne garantit que la liste des indicateurs internationaux de l'efficacité de l'enseignement universitaire ne soit convoquée dans cette perspective.

\subsection{Les « obstacles » à l'usage du concept d'« effet maître »}

Les pages qui précèdent sont illustratives en termes d'obstacles, de résistances et de limites pouvant constituer des freins au développement d'une réflexion sur l'effet maître dans l'université française, même si des pistes d'ouverture se dessinent tout de même. Nous ne reviendrons pas sur ce qui a déjà été évoqué mais nous allons rajouter ici des éléments nouveaux - non exhaustifs - pour apporter des éléments de réponse à la question de l'opportunité, posée dans le titre même de cet article.

La traduction d'un concept issu du champ scolaire à l'enseignement supérieur pose d'abord différents problèmes d'ordre méthodologique. La standardisation des programmes d'enseignement à l'école primaire permet de comparer, toutes choses égales par ailleurs et à partir de données longitudinales le plus souvent, les effets de l'effet maître sur les apprentissages et les performances des élèves confiés à des enseignants différents. Organiser un dispositif équivalent à l'université relève de la gageure, au regard du nombre élevé d'intervenants pédagogiques, de la disparité et de la dispersion des enseignements, de la résistance des enseignants-chercheurs à fonctionner dans un cadre pédagogique harmonisé. L'approche comparative, déterminante pour apprécier l'impact différencié d'un effet maître, reste ainsi en suspens à l'université, au profit d'une restitution d'expériences pédagogiques plutôt individualisées et qui ne possèdent pas toujours une validité des conclusions, interne et externe, à la hauteur des ambitions. Un autre problème méthodologique mérite d'être relevé. Les travaux sur l'effet maître à l'école primaire offrent la possibilité de prendre en considération une diversité d'effets 
(motivation, bien-être, équité, efficacité, etc.). La traduction de ce type de variables, d'ordre "psycho-sociologique » (motivation, bien-être...) ou "socio-politique » (équité, efficacité...) à l'environnement universitaire, est source de difficultés et nécessiterait de prendre des précautions stratégiques et techniques importantes au niveau du recueil des données, de l'interprétation et de la diffusion des résultats des recherches. Rajoutons également le problème méthodologique posé par la comparabilité des expériences de pédagogie universitaire, d'un pays à l'autre (comparabilité des contextes pédagogiques de référence, des concepts utilisés, des outils d'investigation employés...).

On serait ensuite en droit de se demander si l'usage du concept d'« effet maitre » dans la recherche sur la pédagogie universitaire et, de facto, l'armada de termes qui l'accompagnent, ne font pas courir le risque de secondariser l'enseignement universitaire et de l'installer alors dans un " paradigme de simplification » (Morin, 1990), inadapté à la complexité des enjeux dévolus au développement de l'efficacité pédagogique universitaire.

Nous nous référerons enfin à Rege Colet (2006), lorsqu'elle fait un point sur le champ scientifique de la pédagogie universitaire, en s'interrogeant sur la capacité de ce champ encore nouveau à s'inscrire actuellement dans un paradigme scientifique. L'enjeu immédiat ne serait pas de construire une "niche épistémologique» (ibid., p. 219) mais plutôt de réunir une «communauté de praticiens» (p. 217) qui pourront accompagner, scientifiquement et pédagogiquement, le changement dans l'université. Notons également, à l'instar de Musselin (2008, p. 40) que le terme générique d " enseignement " demanderait aujourd'hui à être explicité lorsqu'il s'applique au travail universitaire. Il est utilisé par commodité mais dissimule un nombre de tâches de plus en plus diverses et complexes qui échappent, pour l'instant, à l'observation scientifique et dont le concept d'« effet maître » pourrait justement rendre compte.

\section{Les orientations méthodologiques : l'analyse textuelle d'extraits de la littérature scientifique sur la pédagogie universitaire et sur l'effet maître plus spécifiquement}

Ainsi que nous l'évoquions supra, il ne s'agit pas, dans cet article, d'étudier les pratiques enseignantes universitaires en acte, mais de prendre pour objet les discours tenus sur ces pratiques par les chercheurs ou praticiens-chercheurs. Si la littérature scientifique est rarement exploitée à des fins de validité empirique, nous considérons que cette approche se justifie au regard du projet qui est le nôtre, puisque nous entendons apprécier la place faite aux phénomènes qui relèvent de l'effet maître dans cet espace de production.

Cette perspective nous a conduits à analyser un corpus de plus de trois cents communications présentées par des chercheurs de différentes nationalités, lors de trois récents congrès de l'Association internationale de pédagogie universitaire (AIPU) :

- $24^{\text {ème }}$ congrès de l'AIPU : Vers un changement de culture en enseignement supérieur, regards sur l'innovation, la collaboration et la valorisation (Montréal, Québec, 16-18 mai 2007);

- $25^{\text {ème }}$ congrès de l'AIPU : Le défi de la qualité dans l'enseignement supérieur: vers un changement de paradigme (Montpellier, France, 19-22 mai 2008); 
- $27^{\text {ème }}$ congrès de l'AIPU : Quelle université pour demain ? (Trois-Rivières, Québec, 14- 18 mai 2012).

Au regard du nombre de publications relatives à la pédagogie universitaire, ce corpus ne saurait avoir qu'une valeur indicative. Soulignons toutefois que notre choix s'est fondé sur la compétence reconnue de l'AIPU. Par ses colloques annuels, par sa dimension internationale aussi bien que par sa revue - Revue internationale de pédagogie de l'enseignement supérieur (RIPES) -, l'association constitue en effet un lieu de rencontre, d'expertise et de formation en matière de pédagogie de l'enseignement supérieur. La sélection des $24^{\text {ème }}, 25^{\text {ème }}$ et $27^{\text {ème }}$ colloques répond, quant à elle, à la volonté de permettre au lecteur d'accéder aisément aux textes analysés (textes intégraux ou résumés), ceux-ci se trouvant, pour les trois années retenues, réunis dans des actes publiés sur le web ${ }^{4}$.

L'analyse qualitative des textes sélectionnés, soit près de 4500 pages, avait pour objectif premier d'apprécier et de discuter l'usage fait du concept d'« effet maître » dans la communauté des chercheurs travaillant dans le champ de la pédagogie universitaire. Elle visait, en outre, à spécifier les caractéristiques de cet « effet maître », ou les processus qui s'y apparentent, appliqués à la singularité du contexte universitaire. Afin de ne pas nous limiter à la confirmation prévisible de la rareté des travaux mobilisant ce concept, nous avons souhaité étendre notre étude aux aspects relatifs à l'efficacité ou à la qualité de l'enseignement.

30 Pour ce faire, nous avons procédé en quatre étapes :

1. Un repérage dans l'ensemble du corpus des mentions du terme « effet maître »;

2. La recherche systématique dans l'ensemble du corpus de la mention d'auteurs associés à des recherches portant sur l'effet maître. La liste des onze auteurs a été dressée à partir d'une note de synthèse (Bressoux, 1994), cette source ayant été retenue en raison de sa prééminence dans le champ. Au regard du niveau de positionnement de cette référence à la requête «effet-maître » effectuée sur différents moteurs de recherche ou catalogues de bibliothèque, nous pouvons légitimement la supposer connue d'un chercheur s'intéressant à la question;

3. La constitution d'un corpus restreint: après avoir pris connaissance de l'ensemble des communications, vingt-six articles portant sur une thématique explicitement en lien avec la question de l'efficacité ou de la qualité des enseignements universitaires ont été retenus ;

4. Ce corpus restreint a été soumis à une analyse catégorielle permettant de cartographier les facteurs d'efficacité mis en avant dans les différentes recherches. Ces catégories ont, pour une large part, été établies a priori à partir de la note de synthèse de Bressoux (1994) pour les raisons évoquées au point $\mathrm{n}^{\circ} 2$.

31 Les tableaux ci-après présentent la composition du corpus restreint ainsi que les contextes nationaux dans lesquelles les recherches se sont effectuées :

Tableau 1 : Composition du corpus restreint

\begin{tabular}{|l|l|l|l|l|}
\hline Réf. & Source & Auteurs & Titre de la communication & $\begin{array}{l}\text { Nbre } \\
\text { de } \\
\text { pages }\end{array}$ \\
\hline $07 a$ & $\begin{array}{l}\text { AIPU } \\
2007\end{array}$ & $\begin{array}{l}\text { Béchard, J.-P. \& } \\
\text { Edmond-Péloquin, } \\
\text { L. }\end{array}$ & $\begin{array}{l}\text { Perceptions des étudiants de l'impact d'une } \\
\text { innovation pédagogique authentique sur leurs } \\
\text { apprentissages }\end{array}$ & 3 \\
\hline
\end{tabular}




\begin{tabular}{|c|c|c|c|c|}
\hline $07 \mathrm{~b}$ & $\begin{array}{l}\text { AIPU } \\
2007\end{array}$ & $\begin{array}{l}\text { Raynauld, J. \& } \\
\text { Pelletier, M.-P. }\end{array}$ & $\begin{array}{l}\text { L'efficacité de l'apprentissage en ligne: une } \\
\text { analyse longitudinale d'un cours de politique } \\
\text { économique }\end{array}$ & 3 \\
\hline $07 \mathrm{c}$ & $\begin{array}{l}\text { AIPU } \\
2007\end{array}$ & $\begin{array}{l}\text { Beckers, J. \& } \\
\text { Monville, B. }\end{array}$ & $\begin{array}{l}\text { Former des enseignants efficaces et équitables : un } \\
\text { nouveau défi }\end{array}$ & 3 \\
\hline $07 d$ & $\begin{array}{l}\text { AIPU } \\
2007\end{array}$ & Ghozzi Hamdi, K. & De la pédagogie passive à la pédagogie active & 7 \\
\hline $07 \mathrm{e}$ & $\begin{array}{l}\text { AIPU } \\
2007\end{array}$ & $\begin{array}{lrr}\text { Rigaud, } & \text { D., } & \text { De } \\
\text { Geuser, } & \text { F. } & \& \\
\text { Lanarès, J. } & \end{array}$ & $\begin{array}{l}\text { Ai-je appris quelque chose? Les déterminants du } \\
\text { sentiment d'apprentissage des étudiants à travers } \\
\text { l'évaluation des enseignements à l'Université de } \\
\text { Lausanne }\end{array}$ & 4 \\
\hline $07 f$ & $\begin{array}{l}\text { AIPU } \\
2007\end{array}$ & Paivandi, S. & $\begin{array}{l}\text { La sociologie de l'étudiant et la promotion de la } \\
\text { pédagogie universitaire en France }\end{array}$ & 6 \\
\hline $07 \mathrm{~g}$ & $\begin{array}{l}\text { AIPU } \\
2007\end{array}$ & $\begin{array}{ll}\text { Charles, } & \text { S. } \quad \& \\
\text { Batier, C. } & \end{array}$ & $\begin{array}{l}\text { La visioconférence : un outil efficace pour le suivi à } \\
\text { distance des étudiants }\end{array}$ & 2 \\
\hline $08 \mathrm{a}$ & $\begin{array}{l}\text { AIPU } \\
2008\end{array}$ & $\begin{array}{l}\text { Houart, M. \& } \quad \text { \& } \\
\text { Warzee, N. }\end{array}$ & $\begin{array}{l}\text { La communication pédagogique dans les exposés } \\
\text { de chimie à l'université }\end{array}$ & 11 \\
\hline $08 \mathrm{~b}$ & $\begin{array}{l}\text { AIPU } \\
2008\end{array}$ & Detroz, P. & $\begin{array}{l}\text { L'évaluation des enseignements par les étudiants } \\
\text { paradoxes et défis }\end{array}$ & 17 \\
\hline $08 \mathrm{c}$ & $\begin{array}{l}\text { AIPU } \\
2008\end{array}$ & Condamines, $\mathrm{T}$. & $\begin{array}{l}\text { Le "knowledge management» pour } \\
\text { l'accompagnement à l'entrée dans le métier des } \\
\text { enseignants quand les mondes de l'entreprise et de } \\
\text { l'éducation se rejoignent dans la recherche de la } \\
\text { qualité }\end{array}$ & 12 \\
\hline $08 \mathrm{~d}$ & $\begin{array}{l}\text { AIPU } \\
2008\end{array}$ & Rujanu, M. & $\begin{array}{l}\text { Qualité de l'enseignement et qualité de l'éducation } \\
\text { dans la vision de la pédagogie constructiviste }\end{array}$ & 5 \\
\hline $08 \mathrm{e}$ & $\begin{array}{l}\text { AIPU } \\
2008\end{array}$ & Hadhri, M. & $\begin{array}{l}\text { Améliorer la qualité de l'enseignement supérieur, } \\
\text { réalité, enjeux et perspectives dans le contexte de } \\
\text { l'enseignement supérieur tunisien }\end{array}$ & 14 \\
\hline $08 \mathrm{f}$ & $\begin{array}{l}\text { AIPU } \\
2008\end{array}$ & $\begin{array}{l}\text { Roman, V., Wolfs, } \\
\text { J.-L. \& Robert, F. }\end{array}$ & $\begin{array}{l}\text { Rétroactions, niveaux d'apprentissage et } \\
\text { représentation des connaissances, analyse des } \\
\text { modèles d'apprentissage et d'enseignement }\end{array}$ & 16 \\
\hline $08 \mathrm{~g}$ & $\begin{array}{l}\text { AIPU } \\
2008\end{array}$ & Robert, F. & $\begin{array}{l}\text { Etudiants autonomes et actifs, quel rôle pour } \\
\text { l'enseignant? }\end{array}$ & 14 \\
\hline $08 \mathrm{~h}$ & $\begin{array}{l}\text { AIPU } \\
2008\end{array}$ & $\begin{array}{l}\text { Pelletier, J. \& } \\
\text { Fraser, M. }\end{array}$ & $\begin{array}{l}\text { Un projet de développement des compétences } \\
\text { professionnelles. Réflexion en rapport avec le } \\
\text { curriculum de formation }\end{array}$ & 13 \\
\hline $12 \mathrm{a}$ & $\begin{array}{l}\text { AIPU } \\
2012\end{array}$ & $\begin{array}{l}\text { Mailloux, N. \& } \\
\text { Boyer, J.-C. }\end{array}$ & $\begin{array}{l}\text { L'influence d'une approche basée sur les acquis } \\
\text { expérientiels des étudiantes sur leurs conceptions } \\
\text { au sujet de l'enseignement des } \\
\text { mathématiques au primaire }\end{array}$ & 11 \\
\hline $12 b$ & $\begin{array}{l}\text { AIPU } \\
2012\end{array}$ & $\begin{array}{l}\text { Plumat, J., Baillet, } \\
\text { D., Cobut, B. et al. }\end{array}$ & $\begin{array}{l}\text { Comment favoriser la présence et la participation } \\
\text { active des } \\
\text { étudiants aux dispositifs d'aide à la réussite? }\end{array}$ & 8 \\
\hline
\end{tabular}




\begin{tabular}{|c|c|c|c|c|}
\hline $12 \mathrm{c}$ & $\begin{array}{l}\text { AIPU } \\
2012\end{array}$ & $\begin{array}{l}\text { Dohogne, L. \& } \\
\text { Vanvynck, V. }\end{array}$ & $\begin{array}{l}\text { Expérimenter pour comprendre au travers de l'art } \\
\text { et la science }\end{array}$ & 13 \\
\hline $12 \mathrm{~d}$ & $\begin{array}{l}\text { AIPU } \\
2012\end{array}$ & $\begin{array}{l}\text { Peltier, C. \& } \\
\text { Peraya, D. }\end{array}$ & $\begin{array}{l}\text { Analyse des effets d'une activité réflexive } \\
\text { instrumentée sur la } \\
\text { construction de compétences métacognitives }\end{array}$ & 12 \\
\hline $12 \mathrm{e}$ & $\begin{array}{l}\text { AIPU } \\
2012\end{array}$ & Picard, C. & $\begin{array}{l}\text { Impact de la pédagogie universitaire sur le } \\
\text { développement de } \\
\text { compétences }\end{array}$ & 8 \\
\hline $12 f$ & $\begin{array}{l}\text { AIPU } \\
2012\end{array}$ & $\begin{array}{l}\text { De Vleeschouwer, } \\
\text { M. }\end{array}$ & $\begin{array}{l}\text { Un méta-langage pour aider à la transition } \\
\text { secondaire-université }\end{array}$ & 9 \\
\hline $12 \mathrm{~g}$ & $\begin{array}{l}\text { AIPU } \\
2012\end{array}$ & $\begin{array}{l}\text { Lavigne, G., } \\
\text { Mcanally- } \\
\text { Salas, L. et al. }\end{array}$ & $\begin{array}{l}\text { Moodle et ses registres de navigation, une } \\
\text { information } \\
\text { pédagogique nouvelle }\end{array}$ & 10 \\
\hline $12 \mathrm{~h}$ & $\begin{array}{l}\text { AIPU } \\
2012\end{array}$ & Leduc, $\mathrm{V}$. & $\begin{array}{l}\text { Utilités perçues du Plan de cours ou de la First class } \\
\text { meeting par les étudiants de première et deuxième } \\
\text { années. Quelles informations inclure en priorité } \\
\text { selon eux, et à quelles fins? }\end{array}$ & 10 \\
\hline $12 \mathrm{i}$ & $\begin{array}{l}\text { AIPU } \\
2012\end{array}$ & $\begin{array}{l}\text { Perret, C. \& } \\
\text { Morlaix, S. }\end{array}$ & $\begin{array}{l}\text { Essai de mesure des effets du Plan "Réussite en } \\
\text { Licence" }\end{array}$ & 10 \\
\hline $12 \mathrm{j}$ & $\begin{array}{l}\text { AIPU } \\
2012\end{array}$ & $\begin{array}{lr}\text { Bitar, } & \text { M., } \\
\text { Kaddouri, } & \text { M. } \quad \& \\
\text { Azizi, M. } & \end{array}$ & $\begin{array}{l}\text { La Traduction sur Objectifs Spécifiques } \\
\text { à l'université : Approches didactique } \\
\text { professionnelle en contexte médiatisé }\end{array}$ & 9 \\
\hline $12 \mathrm{k}$ & $\begin{array}{l}\text { AIPU } \\
2012\end{array}$ & $\begin{array}{l}\text { Groleau, A. \& } \\
\text { Fountain, R.-M. }\end{array}$ & $\begin{array}{l}\text { Web } 2.0 \text { = pédagogie } 2.0 ? \text { Un cours universitaire } \\
\text { sous la forme } \\
\text { d'une recherche-action participative }\end{array}$ & 7 \\
\hline \multicolumn{4}{|c|}{26 communications } & 237 \\
\hline
\end{tabular}

Tableau 2 : Contextes nationaux des recherches

\begin{tabular}{|l|l|l|l|l|l|l|l|l|}
\hline & Canada & Belgique & Tunisie & Suisse & France & Roumanie & Mexique & Maroc \\
\hline $\begin{array}{l}\text { Nombre de } \\
\text { communications }\end{array}$ & 6 & 9 & 2 & 2 & 4 & 1 & 1 & 1 \\
\hline
\end{tabular}

\section{L'interprétation des résultats, suite à l'analyse catégorielle de 26 communications portant a priori sur « l'effet maître » à l'université}

\subsection{Le terme d' « effet maître », un absent remarquable}

Les deux premières étapes de notre analyse visaient à apprécier l'influence d'une littérature relative à l'effet maitre dans les contributions présentées aux trois colloques AIPU de 2007, 2008 et 2012. Comme nous l'avions supposé, il ressort de cette première 
phase que le terme d' " effet maître » est faiblement mobilisé. Il n'apparait en tant que tel qu'une seule fois, et encore dans le cadre d'une hypothèse explicative, de façon très allusive et périphérique: "Nous émettons l'hypothèse de la population à $90 \%$ allochtone de cette catégorie. [...] La satisfaction la plus élevée manifestée par les étudiants de la section économique de type court n'est-elle pas due à un effet maitre sachant qu'un seul enseignant a animé les séances de parcours pédagogique professionnel ?» (Cobut \& Boulvain, 2008, p. 979).

Quant à la référence à des publications relevant de recherches ayant trait à l'effet maître, seules trois occurrences apparaissent parmi la liste de onze auteurs que nous avions établie (Averch, Bressoux, Brophy, Doyle, Good, Hanushek, Jacobson, Mingat, Mortimore, Rosenthal \& Veldman) :

- Rosenthal est cité deux fois pour des recherches en lien avec l'effet maitre ;

- Brophy est cité une fois pour des recherches en lien avec l'effet maître.

Nous avons donc confirmation du fait que, malgré des préoccupations proches, ainsi que nous le verrons infra, il y a une étanchéité quasi parfaite entre les travaux de recherche portant sur l'effet maître dans le premier ou le second degré et les travaux qui s'intéressent à la pédagogie universitaire. La relative indétermination du terme de "maître ( (maître d'école, maître de conférences en contexte français) ne suffit probablement pas à dépasser le marquage fort qui associe l'effet maitre à une pédagogie scolaire, expliquant la très faible mobilisation de travaux afférents.

\subsection{Quels facteurs la recherche en pédagogie universitaire privilégie-t-elle lorsqu'elle se préoccupe d'efficacité ?}

Le deuxième objectif de cette étude était d'effectuer, à partir du corpus restreint constitué de communications en lien avec des thématiques ayant une proximité avec l'effet maître, une description des spécificités liées aux contextes de la pédagogie universitaire. Quelles finalités la recherche en pédagogie universitaire poursuit-elle lorsqu'elle étudie l'efficacité des enseignements? Quels objets privilégie-t-elle? Quelles méthodes emploie-t-elle?

Le tableau ci-dessous présente les facteurs d'efficacité retenus et analysés par chacune des 26 communications :

Tableau 3 : Facteurs d'efficacité analysés dans les 26 communications

\begin{tabular}{|l|l|l|}
\hline Facteurs & Références & Total \\
\hline $\begin{array}{l}\text { Les chances d'apprendre (les contenus enseignés correspondent-ils } \\
\text { aux acquisitions évaluées ?) }\end{array}$ & & 0 \\
\hline Le temps & & 0 \\
\hline Taux de réponses exactes aux questions posées par le maître & & 0 \\
\hline Les attentes des enseignants & & 0 \\
\hline Les feedbacks & & \\
\hline Louanges et critiques & & 0 \\
\hline Corrections apportées aux erreurs & & 0 \\
\hline Structuration des activités & & \\
\hline
\end{tabular}




\begin{tabular}{|c|c|c|}
\hline Enseignement dirigé (guidage, planification) & $08 a, 12 c$ & 2 \\
\hline Clarté de l'exposé & $08 \mathrm{a}$ & 1 \\
\hline Questionnement & & 0 \\
\hline Plan de cours & $12 \mathrm{~h}$ & 1 \\
\hline $\begin{array}{l}\text { Dispositifs favorisant l'articulation théorie/pratique dans une } \\
\text { perspective "professionnalisante» }\end{array}$ & $\begin{array}{l}07 a, 07 c, 07 f, 08 c \\
12 e, 12 j\end{array}$ & 6 \\
\hline Réponses des élèves (distribution, modalités) & & 0 \\
\hline $\begin{array}{l}\text { Mobilisation du savoir expérientiel des étudiants (modèle de la } \\
\text { reconstruction du savoir) }\end{array}$ & $12 \mathrm{a}$ & 1 \\
\hline $\begin{array}{l}\text { Approches pédagogiques: enseignement frontal, de groupes, } \\
\text { individualisé, à distance, via les nouvelles technologies } \\
\text { d'information et de communication, les pédagogies actives, les } \\
\text { dispositifs de formation hybrides }\end{array}$ & $\begin{array}{l}07 \mathrm{~b}, 07 \mathrm{~d}, 07 \mathrm{~g}, 08 \mathrm{~d} \\
08 \mathrm{f}, 12 \mathrm{~d}, 12 \mathrm{e}, 12 \mathrm{f} \\
12 \mathrm{~g}, 12 \mathrm{k}\end{array}$ & 10 \\
\hline Valorisation de la combinaison de ces facteurs & $\begin{array}{l}07 \mathrm{e}, 08 \mathrm{~d}, 08 \mathrm{f}, 08 \mathrm{~g}, \\
12 \mathrm{i}\end{array}$ & 5 \\
\hline
\end{tabular}

37 La lecture d'un tel tableau apporte un certain nombre d'informations de nature à préciser notre constat initial. Il apparaît tout d'abord qu'aucune recherche ne s'inscrit dans l'une des cinq premières rubriques du tableau. Ces rubriques sont celles qui concernent le plus étroitement les pratiques réelles d'enseignement nécessitant, à ce titre, des modalités d'investigation spécifiques : observation, filmage, entretiens d'explicitation, etc. Seule la communication $8 \mathrm{a}$ (contexte belge) s'intéresse à la production langagière d'enseignants du supérieur, lors de cours magistraux de chimie et procède par filmage des séances. Aucune des 25 autres communications ne se situe à ce grain d'analyse. Il y a là une notable homogénéité internationale. Les facteurs d'efficacité étudiés relèvent d'organisateurs plus génériques: conceptions pédagogiques, utilisation des nouvelles technologies, mise en place de dispositifs de formation innovants, etc.

Cet état de fait, qui mériterait d'être plus largement établi puis expliqué, contraste avec ce que l'on sait des recherches conduites parallèlement dans le primaire et dans le secondaire, recherches privilégiant généralement l'étude in situ des interactions maitreélèves. Il paraît intéressant de souligner qu'une grande part des recherches présentées porte sur des contextes dans lesquels le chercheur s'est trouvé impliqué, soit comme enseignant, soit comme étudiant. Cette implication est parfois clairement posée : « Avionsnous atteint l'objectif de créer un environnement pédagogique de qualité? 》 (07a), "C'est dans ce cadre que nous nous permettons de présenter notre expérience: 11 ans dans le domaine de l'enseignement des techniques de commercialisation (TC), basé essentiellement sur les méthodes actives d'apprentissage» (07d), "Lors de cette communication, nous souhaitons partager et présenter une expérience singulière mise en place début 2011 dans notre établissement par une artiste et une scientifique, toutes deux enseignantes » (12c). Elle est parfois plus implicite et peut être déduite en croisant diverses informations présentes dans l'article: statut de l'auteur, discipline enseignée, nature du cours, établissement, etc. Dès lors, les recherches s'inscrivent, pour une grande part, dans une préoccupation d'auto-évaluation des enseignements mis en œuvre. Une double finalité les anime: a) la régulation des 
enseignements réalisés souvent par les chercheurs eux-mêmes, b) la promotion d'innovations pédagogiques dans la communauté des praticiens.

Un tel tableau, s'il ne remet nullement en cause la scientificité des travaux, pourrait conduire à s'interroger sur les effets de la promotion visée. La grande hétérogénéité des contextes ne réduit-elle pas considérablement la transférabilité des résultats? Le choix d'étudier des contextes locaux, à forte spécificité, tiendrait-il à la difficulté d'enquêter sur des terrains auxquels le chercheur n'a pas un accès direct?

Partageant avec les recherches que nous venons d'évoquer le même caractère d'implication, un nombre conséquent de travaux s'inscrivent dans des préoccupations plus transversales, s'attachant aux thématiques suivantes :

- L'articulation théorie/pratiques dans une perspective de professionnalisation des étudiants ;

- La place grandissante des nouvelles technologies d'information et de communication dans l'enseignement supérieur et leur apport ;

- L'aggiornamento pédagogique : i.e. le passage d'une pédagogique transmissive dont le cours magistral est l'archétype à des pédagogies de type actives ou socioconstructivistes.

Seize communications sur vingt-six portent sur l'une de ces trois thématiques. Cela n'a rien de surprenant, puisque celles-ci rejoignent peu ou prou les grandes évolutions que l'université a eu à connaître : la professionnalisation des formations, la massification, la révolution numérique. La perspective adoptée dans ces différentes communications relève d'une volonté d'opérationnalisation et d'optimisation. L'objectif assumé n'est pas d'effectuer une analyse critique de ces processus: "Notre intention est de faire vivre aux étudiants une activité professionnelle authentique, qui se rapproche le plus du métier d'un dirigeant lorsqu'il assiste à une foire commerciale dans son domaine. De l'avis de tous, cette journée fut un véritable succès médiatique. » (07a), "Ces résultats laissent présager que l'enseignement de l'économie en ligne est une option à considérer sérieusement dans la mesure où les bénéfices sont plus grands que les coûts!» (07b), "Étant donné le succès de cette méthode pédagogique active et participative, l'expérience est en train d'être généralisée au sein de notre réseau; toutefois son application reste tributaire de la volonté des enseignants qui y croient vraiment et qui sont prêts à s'y impliquer " (07d), "Nous partageons, dans cette communication, l'expérience de recherche et d'apprentissage que nous avons vécue avec vingt-deux autres étudiants et étudiantes dans le cadre d'un cours qui a pris la forme d'une recherche-action participative et qui s'est appuyé sur une approche socioconstructiviste» (12k).

À la lecture des différentes communications, une question émerge, qui demeure irrésolue : qu'en est-il de la conception du «maître » que l'on se fait à l'université, de l'importance que l'on accorde à son rapport singulier au savoir, à l'impact de sa personnalité sur le développement intellectuel des étudiants? Les trois évolutions que nous venons d'évoquer (professionnalisation, nouvelles technologies, révolution pédagogique) ont ceci de semblables qu'elles pourraient conduire à une minoration du rôle du "maître ", c'est-à-dire celui qui se trouve au centre, qui incarne une pensée originale et donner à voir par l'épreuve du discours l'exercice de cette pensée. Ne peut-on penser qu'à l'université, outre l'acquisition de savoirs et des savoir-faire, l'étudiant est à la recherche de figures identificatoires, de visions originales? L'enseignant-chercheur pourrait être envisagé dans cette perspective, justement ou indûment, comme un maître à penser, voire comme un modèle? Evoquons à titre d'exemple la façon dont une professeure de lettres se souvient de ses étudiants : «Ils voulaient réinventer l'amour et venaient là, naïvement, à la recherche de paroles viatiques inaltérables à l'usure du temps, ces paroles qu'elle tremblait alors de ne pas savoir communiquer en pénétrant 
dans le grand amphi noir, bourdonnant, où ils se taisaient soudain, attentifs et exigeants, aussi prompts à contester qu'à adorer. » (Plouvier, 1998, pp.14-15). Cette position est discutable; il n'était pas dans le projet des communications analysées d'ouvrir un débat sur le rôle du maître à l'université, mais ne peut-on supposer sur ce point une différence notable entre les conceptions que l'on peut en avoir dans le secondaire ou dans les études supérieures?

\subsection{Quelles méthodes d'investigation ont été privilégiées ?}

Poursuivons à présent la description entreprise en nous intéressant aux méthodes qui sont privilégiées par les différentes recherches. Les deux tableaux qui suivent en exposent les caractéristiques principales :

Tableau 4 : Type de recherches conduites

\begin{tabular}{|l|l|l|}
\hline Type & Références & Total \\
\hline Comparative & $07 \mathrm{~b}, 07 \mathrm{e}, 08 \mathrm{a}, 12 \mathrm{~h}, 12 \mathrm{i}$ & 5 \\
\hline Longitudinale & $07 \mathrm{c}$ & 1 \\
\hline Etude de cas & $\begin{array}{l}07 \mathrm{a}, 07 \mathrm{c}, 07 \mathrm{~d}, 07 \mathrm{~g}, 08 \mathrm{c}, 08 \mathrm{~g}, 12 \mathrm{a}, 12 \mathrm{c}, 12 \mathrm{~d}, 12 \mathrm{e}, \\
12 \mathrm{~g}, 12 \mathrm{j}, 12 \mathrm{k}\end{array}$ & 13 \\
\hline $\begin{array}{l}\text { Méta-analyse (à partir de travaux de } \\
\text { recherche) }\end{array}$ & $07 \mathrm{f}, 08 \mathrm{~b}, 08 \mathrm{e}$ & 3 \\
\hline Réflexion générale & $08 \mathrm{f}$ & 1 \\
\hline
\end{tabular}

Tableau 5 : Méthodologie employée pour la mesure des effets

\begin{tabular}{|l|l|l|}
\hline Méthodes & Références & Total \\
\hline Questionnaire étudiant & $\begin{array}{l}07 \mathrm{a}, 07 \mathrm{e}, 08 \mathrm{a}, 08 \mathrm{~b}, 08 \mathrm{~g}, 07 \mathrm{c}, 12 \mathrm{a}, \\
12 \mathrm{~h}, 12 \mathrm{j}\end{array}$ & 9 \\
\hline $\begin{array}{l}\text { Analyse de journaux de bord et de rapports réflexifs } \\
\text { d'étudiants }\end{array}$ & $12 \mathrm{~d}$ & 1 \\
\hline $\begin{array}{l}\text { Evaluation des performances des étudiants mobilisant } \\
\text { des modèles de régression }\end{array}$ & $07 \mathrm{~b}, 12 \mathrm{i}$ & 2 \\
\hline $\begin{array}{l}\text { Analyse statistique des connexions à une plateforme } \\
\text { MOODLE et effet sur les notes }\end{array}$ & $12 \mathrm{~g}$ & 1 \\
\hline Non exposée & $\begin{array}{l}07 \mathrm{~d}, 07 \mathrm{f}, 07 \mathrm{~g}, 08 \mathrm{c}, 08 \mathrm{~d}, 08 \mathrm{e}, 08 \mathrm{f}, \\
12 \mathrm{c}, 12 \mathrm{e}, 12 \mathrm{f}, 12 \mathrm{k}\end{array}$ & 11 \\
\hline
\end{tabular}

En adéquation avec le caractère local des thématiques étudiées, il apparaît que la moitié des recherches conduites consiste en des études de cas. Elles adoptent une visée descriptive dans laquelle la validation statistique des résultats n'est pas recherchée. Les recherches menées en contexte français ne présentent pas d'originalité remarquable au regard de cette situation. Les méthodes permettant d'attester des effets positifs des dispositifs sur les apprentissages des étudiants ne sont pas systématiquement détaillées 
(11 communications ne les précisent pas). L'effet maître apparaît comme un postulat dont on ne cherche pas à établir comparativement ou quantitativement la pertinence: l'objectif est davantage de présenter les choix de dispositifs ou de pédagogie qui sont censés y contribuer.

Il est à noter que l'évaluation des performances des étudiants reste peu mobilisée (2 cas). Les questionnaires étudiants constituent un moyen de confirmation bien plus important (9), que ce soit afin de recueillir le point de vue de ces étudiants ou pour apprécier l'évolution de leurs conceptions. Notons que les recherches qui adoptent une démarche statistique concluent à l'impossibilité d'avérer un effet de l'enseignement sur l'apprentissage : "Les nombres totaux de clics et de connexions sont fortement corrélés [...] mais ni l'une, ni l'autre ne l'est avec les notes finales. Ceci laisse entendre que les étudiants soit suivent des stratégies divergentes, soit obtiennent des résultats différents avec une même stratégie. Ainsi, à titre d'exemple, les deux étudiants avec le plus de clics et de connexions ont obtenu, pour l'un une note maximale, pour l'autre la deuxième plus faible. » (12g), "Les résultats sont décevants, que ce soit au niveau de l'effet global du plan de réussite appréhendé par des variables muettes sur l'ensemble de l'échantillon (modèles 1) ou filière par filières (modèles 2). Les effets ne semblent pas très significatifs et encore moins massifs. » (12i). Ainsi que l'évoque l'un des auteurs : "Il faut espérer que ce type de recherche se généralise car avant que puissent se dégager des conclusions quelque peu généralisables, il faudra démultiplier les études en fonction des matières, des filières, des niveaux d'études, des cheminements. " (12g). La fragilité des résultats et leur impossible généralisation apparaissent comme une conséquence prévisible des choix scientifiques et méthodologiques opérés. Elles conduisent en l'état à une grande prudence quant à l'usage qui pourrait être fait de facteurs relevant de l'effet maître, dans l'explication des performances des étudiants.

\section{Conclusion}

Interroger, comme nous l'avons fait, les recherches en pédagogie universitaire sous l'angle du rôle que peut y jouer l'effet maître peut paraître tenir d'un exercice formel, dès lors que ceux qui s'y livrent sont des enseignants-chercheurs en sciences de l'éducation, censément au fait de l'importance toute relative accordée au versant pédagogique de leur pratique. Est-ce à dire qu'en se proposant d'apprécier la "notoriété » d'un concept issu $\mathrm{du}$ primaire et du secondaire, cette contribution ne pouvait que confirmer une ignorance manifeste, à défaut d'être cultivée ? Les communications analysées, si elles confirment l'absence quasi-unanime de références au terme d' " effet-maître », démontrent toutefois en la matière une proximité plus grande que celle à laquelle nous aurions pu nous attendre. La remise en cause du modèle transmissif ainsi que la promotion d'une pédagogie active apparaissent de façon récurrente. À la différence des travaux conduits dans le champ scolaire, une telle orientation peine à trouver ses arguments dans l'analyse des pratiques réelles d'enseignement. La salle de classe paraît, à ce jour, plus ouverte au chercheur que ne le sont l'amphithéâtre, la salle de travaux-dirigés, ou le bureau du directeur de mémoire.

Nous l'avons vu, c'est dans une configuration singulière que la notion d' " effet maître » émerge de notre analyse, amputée de la part la plus contextualisée de ses attributs. Aucune des recherches composant notre corpus ne s'est penchée sur la cohérence des contenus enseignés et des acquis évalués, sur le temps effectivement consacré aux apprentissages, sur l'explicitation des attentes des enseignants-chercheurs, sur l'usage 
des feedbacks, des louanges ou des corrections apportées aux erreurs des apprenants. Bien que plusieurs recherches mobilisent des méthodologies fines d'évaluation des acquis (modèles de régression, analyse de journaux de bord et de rapports réflexifs d'étudiants), le lien entre ces résultats et les pratiques pédagogiques des enseignants-chercheurs n'est pas étudié.

Que retenir d'un tel constat? En développant des problématiques oublieuses de l'effetmaitre, la recherche en pédagogie universitaire ne risque-t-elle pas de manquer des aspects stratégiques pour la profession? L'éventualité d'un pilotage centré sur les acquis des étudiants ou leur insertion, conduisant à une remise en cause de pratiques magistrales propres à l'université, est-il à redouter? Le développement de nouvelles technologies, qui permettent la mise à disposition de contenus de cours sur des plateformes diverses, pourrait-il se conjuguer avec l'essor de pédagogies issues de modèles socioconstructivistes pour accomplir une «secondarisation" redoutée de l'enseignement supérieur? Ces questions cèdent sans nul doute le pas à d'autres plus centrales pour l'avenir des Universités. À défaut de répondre à une préoccupation vive, le développement de recherche sur l'effet-maître pourrait avoir pour mérite de contribuer à préciser le rôle des enseignants-chercheurs, dans une institution qui peine parfois à se déterminer sur le sens de la double injonction qui lui est faite : professionnaliser tout en se professionnalisant.

\section{BIBLIOGRAPHIE}

Adangnikou, N. (2008). Peut-on parler de recherche en pédagogie universitaire, aujourd'hui, en France ? Revue des sciences de l'éducation, 34(3), 601-621.

Albero, B. (Dir.) (2003). Autoformation et enseignement supérieur. Paris : Lavoisier.

Annoot, E., \& Fave-Bonnet, M.-F. (Dir.) (2004). Pratiques pédagogiques dans l'enseignement supérieur : enseigner, apprendre, évaluer. Paris : L'Harmattan.

Bart, D. (2008). Les modes de valorisation de la recherche en Sciences de l'éducation et le développement professionnel des enseignants-chercheurs de la discipline (Thèse de doctorat en sciences de l'éducation sous la direction d'A. Jorro et le tutorat de V. Bedin, non publiée, soutenue le 04 décembre 2008). Université de Toulouse-Le Mirail, Toulouse, France.

Bedin, V., \& Saint-Jean, M. (2013). L'université française face aux défis de sa propre réforme : vers des crises ou des changements? In V. Bedin (Ed.), Conduite et accompagnement du changement. Contribution des sciences de l'éducation (ouvrage à paraître). Paris : L'Harmattan.

Bedin, V. (2012). L'évaluation des enseignants-chercheurs en France. Spirale, 49, 157-172.

Bedin, V., \& Broussal, D. (2011). L'évaluation interactive des apprentissages dans l'enseignement supérieur : intérêt pédagogique et limites. Les Dossiers des Sciences de l'Education, 26, 133-144.

Behrens, M. (Dir.) (2007). La qualité en éducation. Pour réfléchir à la formation de demain. Québec : Presses Universitaires du Québec. 
Bouchard, C., \& Plante, J. (2002). La qualité : mieux la définir pour mieux la mesurer. Les Cahiers du Service de pédagogie expérimentale, 11-12, 219-236.

Boyer, E. L. (1990). Scholarship Reconsidered: Priorities of the Professoriate. Princeton, NJ: Carnegie Foundation for the Advancement of Teaching.

Bressoux, P. (1994). Les recherches sur les effets-écoles et les effets-maîtres. Note de Synthèse. Revue française de pédagogie, 108, 91-137.

Bressoux, P. (2001). Réflexions sur l'effet-maître et l'étude des pratiques enseignantes. Les Dossiers des Sciences de l'Education, 5, 35-52.

Bru, M. (2004). Pratiques enseignantes à l'université. Opportunité et intérêt des recherches. In E. Annoot \& M.-F. Fave-Bonnet (Eds.), Pratiques pédagogiques dans l'enseignement supérieur : enseigner, apprendre, évaluer (pp. 17-36). Paris : L'Harmattan.

Cobut, B., \& Boulvain, M. (2008). Un dispositif obligatoire de soutien aux étudiants de 1 ère BA à la haute école Francisco Ferrer à Bruxelles, des ailes pour réussir ! Actes du $25^{\text {ème }}$ congrès de l'AIPU : Le défi de la qualité dans l'enseignement supérieur : vers un changement de paradigme (Montpellier, France, 19-22 mai 2008), 969-981, URL : http://www.aipu2008-montpellier.fr/index.php? dossier_nav=839\&action=0\&id =, accédé le 22 août 2012 .

Dejean, J. (2002). L'évaluation de l'enseignement dans les universités françaises. Rapport établi à la demande du Haut conseil de l'évaluation de l'école. Paris : HCEE. Disponible sur Internet : http:// cisad.adc.education.fr/hcee/documents/rapport5journalistes.pdf.

De Ketele, J.-M. (2010). La pédagogie universitaire : un courant en plein développement. Revue française de pédagogie, 172, 5-13.

Fave-Bonnet, M.-F. (2003). L'évaluation dans les universités en Europe : une décennie de changements. Revue des Sciences de l'Education, 29, 2, 319-336.

Langevin, L. (2009). Accompagnement pédagogique : une expertise à développer. In D. Bédard, \& J.-P. Béchard (Eds.), Innover dans l'enseignement supérieur (pp. 140-150). Paris : PUF.

Morin, E. (1990). Science avec conscience. Paris : Le Points.

Musselin, C. (2008). Les universitaires. Paris : Éditions La Découverte.

Plouvier, P. (1998). Solitudes ordinaires. Paris : L'Harmattan.

Rege Colet, N. (2006). La pratique enseignante : un nouveau paradigme ou une communauté de praticiens ? In M. Romainville \& N. Rege Colet (Dir.), La pratique enseignante en mutation à l'université (pp. 217-226). Bruxelles : De Boeck.

Rege Colet, N., McAlpine, L., Fanghanel, J., \& Weston, C. (à paraître). Le concept de Scholarship of Teaching and Learning. Recherche \& Formation, 67.

Romainville, M. (2009). Entre l'écorce et l'arbre. Principes et modalités de la formation pédagogique des enseignants universitaires. In V. Bedin (Dir.), L'évaluation à l'université. Évaluer ou conseiller? (pp. 263-273). Rennes : Presses universitaires de Rennes.

Romainville, M. (2002). L'évaluation des acquis dans l'enseignement universitaire. Rapport établi à la demande du Haut conseil de l'évaluation de l'école. Paris : Haut conseil de l'évaluation de l'école. Disponible sur Internet : http://cisad.adc.education.fr/hcee.

Saroyan, A., Amundsen, C., McAlpine, L., Weston, C., Winer, L., \& Gandell, T. (2006). Un modèle de développement pédagogique pour l'enseignement universitaire? In M. Romainville \& N. Rege Colet (Dir.), La pratique enseignante en mutation à l'université (pp. 171-184). Bruxelles : De Boeck. 
Stengers, I. \& Schlanger J. (Dir.) (1989). Les concepts scientifiques. Invention et pouvoir. Paris : Editions La Découverte.

Talbot, L. (2008). Etudier les pratiques d'enseignement. Un exemple comparatif au collège et à l'école primaire. Les Dossiers des Sciences de l'Education, 19, 81-101.

Talbot, L. (2011). Prendre en compte la diversité des élèves. Ressource ou défi pour les enseignants? Les Dossiers des Sciences de l'Education, 26, 7-12.

Viry, L. (2006). Le monde vécu des universitaires ou La République des Egos. Rennes : Presses Universitaires de Rennes.

\section{NOTES}

1. Cette enquête a été coordonnée par la commission "Cursus, publics, labos » de l'Association des enseignants et chercheurs en sciences de l'éducation (AECSE, www.aecse.net, France). Les premiers résultats ont été présentés par Yvan Abernot en janvier 2011 lors de l'assemblée générale de cette association.

2. Le groupe de réflexion "Revues " de l'Association des enseignants et chercheurs en sciences de l'éducation (AECSE, www.aecse.net), coordonné par Françoise Laot, a élaboré une cartographie des revues scientifiques du champ des sciences de l'éducation en France. Une partie du travail réalisé a été présenté en janvier 2011 lors de l'assemblée générale de cette association.

3. Leurs travaux de recherche concernent le contexte universitaire et sont référencés dans la bibliographie de cette contribution.

4. AIPU 2007 : http://aipu2007.umontreal.ca/pdf/actes_detailles.pdf, accédé le 22 août 2012 ;

AIPU 2008 : http://www.aipu2008-montpellier.fr/index.php?dossier_nav=839\&action=0\&id=, accédé le 22 août 2012 ;

AIPU 2012: https://oraprdnt.uqtr.uquebec.ca/pls/public/gscw031? owa_no_site $=2220 \&$ owa_no_fiche $=47$ \&owa_apercu=N\&owa_imprimable $=$ N\&owa_bottin $=\quad$ (accédé le 22 août 2012).

\section{RÉSUMÉS}

Cet article questionne la place et l'opportunité des travaux sur l'effet maître dans les recherches en pédagogie universitaire. Il interroge plus largement l'efficacité de l'enseignement dans ce contexte. La notion d'«effet maître" reste marginale dans la recherche en pédagogie universitaire, elle-même peu développée en sciences de l'éducation en France. Le contenu de l'article explore donc des points aveugles et des zones d'ombre. La méthode qui a été empruntée a consisté à analyser des textes de communications effectuées dans le cadre de colloques de l'Association internationale de pédagogie universitaire (AIPU), pour y repérer des indicateurs éventuels de l'effet maître. À partir de cette focale d'analyse, une réflexion est conduite sur l'évolution de la pédagogie universitaire.

This article is interested in importance and opportuneness of studies concerning teacher effects in university pedagogy. The authors examine more widely teaching effectiveness in this context. 
In university pedagogical research, the notion of "teacher effect" is still marginal. In the same way, the university pedagogical research is not very developed in French educational sciences. The contents of the article thus investigate blind spots and shadow zones. The method consisted in analyzing texts of communications made during congress of the International association of university pedagogy (AIPU). The authors sought to locate possible indicators of teacher effects. With this analysis prospect, a reflection is led on the evolution of the university pedagogy.

INDEX

Mots-clés : effet maître, efficacité, université, pédagogie universitaire, recherches en éducation Keywords : teacher effects, effectiveness, university pedagogy, research in education

\section{AUTEURS}

\section{VÉRONIQUE BEDIN}

Département sciences de l'éducation et de la formation, UMR « Education Formation Travail Savoirs » (EFTS), Université de Toulouse II-Le Mirail (France)

DOMINIQUE BROUSSAL

Département sciences de l'éducation et de la formation, UMR « Education Formation Travail Savoirs » (EFTS), Université de Toulouse II-Le Mirail (France) 